\title{
Solutions for Unexpected Challenges Encountered when Integrating Research Genomics Results into the EHR
}

\author{
Luke V. Rasmussen ${ }^{1}$ Christin Hoell ${ }^{2}$ Maureen E. Smith ${ }^{2}$ Rex Chisholm ${ }^{2}$ Justin Starren ${ }^{1}$ \\ Sharon Aufox ${ }^{2}$ Yuan Luo ${ }^{1}$ Laura J. Rasmussen-Torvik ${ }^{1}$ \\ ${ }^{1}$ Department of Preventive Medicine, Northwestern University \\ Feinberg School of Medicine, Chicago, Illinois, United States \\ ${ }^{2}$ Center for Genetic Medicine, Northwestern University Feinberg \\ School of Medicine, Chicago, Illinois, United States \\ Address for correspondence Luke V. Rasmussen, MS, Department o \\ Preventive Medicine, Northwestern University Feinberg School of \\ Medicine, 11th Floor Rubloff Building, 750 North Lake Shore Drive, \\ Chicago, IL 60611, United States \\ (e-mail: luke.rasmussen@northwestern.edu).
}

ACI Open 2020;4:e132-e135.

\begin{abstract}
Keywords

- electronic health record

- genetics

- precision medicine

Background While there have been published reports detailing technical challenges of incorporating genetic test results into the electronic health record (EHR) with proposed solutions, less has been published about unanticipated sociotechnological or practical communication challenges involved in this process.

Objectives This study was aimed to describe unanticipated issues that arose returning genetic research results through the EHR as part of the National Human Genome Research Institute (NHGRI)-funded electronic Medical Records and Genomics (eMERGE) 3 consortium, and provide lessons learned for future implementations Methods We sequenced 3,000 participants on a 109-gene panel and returned genetic results initially in person and/or by letter, with a later release directly into the EHR and patient portal.

Results When results were returned through the EHR, multiple participants expressed confusion and contacted the health system, resulting in our institution temporarily freezing our return of research results.

Discussion We determined the likely causes of this issue to be (1) the delay between enrollment and results return, (2) inability to personalize mass e-mail messages announcing new research test results in the EHR, (3) limited space for description of test results in the EHR, and (4) the requirement to list an ordering physician for research results in the EHR. For future return of results, we propose sending preparatory e-mails to participants, including screenshots of how they can expect to see their results presented in the EHR portal.

Conclusion We hope our lessons learned can provide helpful guidance to other sites implementing research genetic results into the EHR and can encourage EHR developers to incorporate greater flexibility in the future.
\end{abstract}

received

April 1, 2020

accepted after revision

September 18, 2020
DOI https://doi.org/

10.1055/s-0040-1719059.

ISSN 2566-9346. (c) 2020 Georg Thieme Verlag KG Stuttgart · New York
License terms

(ब)(1) 


\section{Background and Significance}

The process of incorporating genetic test results into the electronic health record (EHR) is challenging and can be further complicated when conducted as part of a research study where integration is required with unfamiliar clinical processes. While there have been published reports about the purely technical challenges and solutions, ${ }^{1-4}$ less has been published about unanticipated sociotechnological or practical communication challenges involved in this process. Identifying and disseminating lessons learned is a critical aspect of improving the design and implementation of health information technology, ${ }^{5}$ and may assist others embarking on genetic implementation projects to achieve a smoother integration of genetic test results into the EHR.

In this case report, we describe our approach to return individual genetic research results to patients and their physicians through the EHR as part of the third phase of the National Human Genome Research Institute (NHGRI)funded electronic Medical Records and Genomics (eMERGE) consortium. We present unanticipated issues that arose and suggest specific steps that other clinical sites take in future implementations to avoid some of the issues encountered by our team. We also suggest potential future design changes for subsequent versions of EHR systems to allow greater flexibility in the return of genetic research results.

\section{Methods}

Within eMERGE 3, institutions were paired with one of two laboratories, Baylor College of Medicine Human Genome Sequencing Center (HGSC) and Partners HealthCare Laboratory for Molecular Medicine partnered with the Broad Institute (Partners). Blood samples were tested using a set panel of 109 genes and 1,551 single-nucleotide variant (SNV) sites and processed in a Clinical Laboratory Improvement Amendments (CLIA)-compliant manner using a harmonized clinical sequencing pipeline at each laboratory. ${ }^{6}$ The sequencing results from each laboratory were then returned to sites in the same standard format as XML results with associated PDF interpretations. ${ }^{7}$ Northwestern partnered with HGSC and established a secure download to receive results.

In a previous phase of eMERGE, we developed an Ancillary Genomics System (AGS), ${ }^{2}$ which allowed us to take struc- tured results from an external laboratory, perform normalization and translation steps, and convert the results into a structured observation sent to our Epic EHR (Epic Systems, Verona, Wisconsin, United States). We extended the functionality of the AGS for eMERGE 3 to process the XML results provided by the HGSC, store the specific variant(s) that were identified for a patient (if any were found), and prepare a final result as a structured laboratory panel. One novel aspect of our approach was that we were able to incorporate participant preferences around the types of results they wanted to receive. For example, if a participant indicated they did not wish to receive results related to cancer, and a pathogenic variant for a cancer-related condition was identified, the AGS would suppress that finding altogether, blinding the study team, patient, and clinical team from the result. A "break the glass" option was built into the AGS to allow the study team to review the original results, if necessary.

-Fig. 1 shows the high-level flow of data for the return of results. The results were reviewed within the AGS by a genetic counselor on the study, and two formats were prepared: a highly visual, formatted version used for printing and mailing, and a second plain text representation used for placement in the EHR. When released to the EHR, the results were transmitted as a Health Level Seven (HL7) v2 message. Because of the institutional workflow used for provisioning and acquiring biological samples for the study, the results are returned and displayed in the EHR but have no corresponding order record associated with them. Multiple dates were associated with each result (i.e., date of specimen collection and date processed by HGSC), but only one date would be displayed in the EHR. We chose the date that the result was released to the EHR as the display date, as a historical date may have caused the result to get lost with the acquisition of more recent results. In addition, the HL7 message required an institutional physician to be named as the requesting provider. We chose a coinvestigator who is a practicing human geneticist and cardiologist, as she was established within the system, although she did not routinely interact with patients receiving negative or noncardiac results. These results were filed within the EHR alongside other laboratory results, which allowed us to utilize existing EHR capabilities for their use and management. Our results were configured to be automatically released to the patient portal (MyChart) 7 days after they were transmitted to Epic, and patients received a generic e-mail indicating they had new

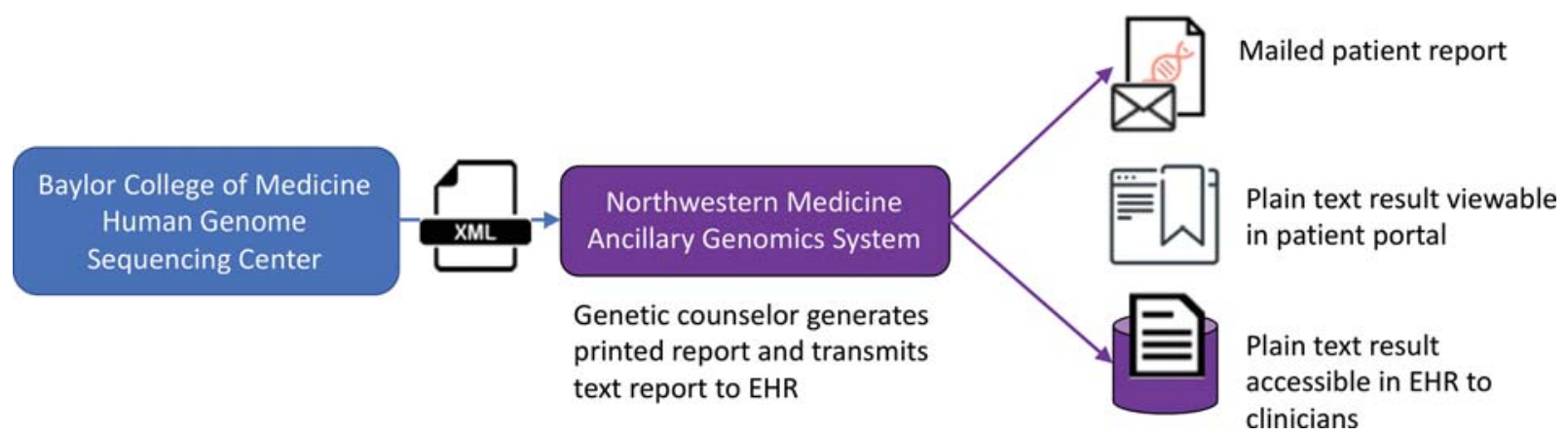

Fig. 1 Flow of genetic results from the sequencing center (Baylor) to Northwestern Medicine. Multiple modalities were available for physicians and patients to access the results. EHR, electronic health record. 
results available in MyChart. The results accessed from MyChart, because they were the plain text HL7 messages, had different formatting from the mailed letter that the patient had received. The interpretation and recommendations, however, were identical.

\section{Results}

Enrollment for eMERGE 3 began at Northwestern on December 18,2015 , and was completed on May 8, 2018. Genetic counselors returned "positive" results (i.e., pathogenic variants, likely pathogenic variants, or risk alleles) in person or by phone between December 11, 2017 and June 26, 2019. Negative results were returned in the same timeframe via letters mailed directly to participants (two individuals received results solely via e-mail). The mean time between date of consent and return of results in-person, via phone, or via mail was 526 days ( $S D=85.7$, range: $81-963$ days). The electronic release of results into the EHR took place July 9, 2019 through August 30, 2019.

\section{Findings}

Recognizing the lag between consent, initial return of results (in-person/phone/mail), and electronic placement of results in the EHR, the study team attempted to communicate with participants about the return of results process; an e-mail was sent to participants 1 week before EHR deposit to alert them they would be receiving a MyChart message indicating genetic research results were available, and that these results were the same as the results sent earlier by mail. Initially, investigators explored whether it would be possible to customize the patient e-mail generated when test results are placed in the EHR; however, because of local software and Health Insurance Portability and Accountability Act (HIPAA) limitations, such customization was not possible. Therefore, when research genetic results were deposited in the EHR, study participants were notified with the standard generic email indicating a new test result was available in MyChart.

Shortly after the release of genetic results into the EHR, multiple study participants contacted our health system about "unexpected test results" appearing in MyChart; many expressed concern that they may be being billed inappropriately for tests, or were receiving someone else's medical information. No formal evaluation of the return of results process was conducted, but in interactions with the study team, participants reported initial confusion viewing an unfamiliar test name in MyChart ("eMERGE-Seq Panel," whereas they consented to "The Genetic Testing and Your Health Study"), as well as an ordering physician who was unfamiliar to the most of patients. While not explicitly stated, we also surmise confusion due to the fact that the plain text formatted version of the report differed visually from their mailed copy. Had the participants read the linked test results in detail, they would have received extensive information about eMERGE 3 , interpretation of the test results, and appropriate study contact numbers to use in the event of any questions. At least three individuals reached out to the ordering physician listed on the test result (either directly or via their primary care doctor) and were appropriately directed to the eMERGE 3 study staff to address their concerns. One individual contacted patient services, and then was directed to risk management who began the process of removing the results (classified as a misdirected laboratory report) from the EHR portal before contacting the ordering physician. A second participant contacted patient services with a similar concern; this concern was elevated to multiple offices within the health system over a 12-day period before finally being brought to the attention of the ordering physician and study staff. During this 12-day period, the health system decided to remove all similar eMERGE 3 test results from the patient portal view, although they remained in the EHR. The process to make the results available again in MyChart required internal discussion with our health system's EHR team, approval from a clinical review committee, and a process of approximately 8.5 weeks in which our local EHR team engaged the EHR vendor to determine the appropriate way to rerelease the results.

\section{Discussion}

We believe these unexpected challenges in the return of research genetic results arose because of four distinct issues as follows: (1) the length of time between enrollment, result return to participants, and placement in the EHR; (2) inability to personalize EHR e-mail messages announcing new test results in the EHR; (3) the limited space for description of the test results in the EHR; and (4) the requirement to list a specific ordering physician for all results including research results. As a result of lessons learned from this experience, we have identified strategies to address these issues that we recommend other institutions consider when implementing the placement of genetic research results into the EHR.

Participant recall can vary (especially over time) about the details of participation in research studies. ${ }^{8,9}$ As we anticipate delays in future studies between enrollment of patients and the return of results, we propose low-tech solutions that may be employed in the future return of results. These solutions require no systemic changes in our EHR, as changes to the EHR generally involve a great deal of time, resources, and multiple layers of permissions, often not feasible to achieve in the time span and budget of a typical research study. First, we propose that 48 hours before releasing results to a participant's EHR, we will e-mail the participant a letter, explaining that they will receive a generic e-mail from Northwestern Medicine about having a new test result available for viewing and explain that this test result is from their participation in a specific study. The letter should contain a screenshot of the generic results email, and a screenshot of how the study test results will appear in the participant's list of test results in the portal, for further emphasis. Second, for future studies, we will ask that any participants with a MyChart account provide the e-mail linked to their MyChart account to study staff for purposes of study communication; we believe in some cases our preparatory e-mail sent before return of eMERGE 3 results was not effective because it went to a different e-mail account than the EHR notification e-mail. Third, we propose using the word 
"RESEARCH" in the test name displayed for the study related test results in the EHR portal, and have implemented this retrospectively for our eMERGE 3 results. We believe this will better associate the result with this research study in participants' minds, and we hope that this will avoid confusion for anyone not receiving or not reading the preparatory e-mail.

In addition to the low-tech solutions described above, we recommend that EHR developers consider adding flexibility to EHR software to permit customization in the return of research results. One solution would be to create an e-mail template for test result return that reads "You have a new RESEARCH test result. Please logon to the EHR portal for more information." A second solution would be to permit the listing of a research study (or a doctor on behalf of a research study) under "ordering physician" in the EHR.

Finally, this experience has allowed us to strengthen ties between research and clinical operations at our organization. Recently, a clinical Research \& Development (R\&D) team was developed that specifically liaises between research and clinical teams which will be beneficial in future projects.

\section{Conclusion}

While returning research genetic test results to the EHR at our institution, we ran into unanticipated challenges due to participants confusing research test results with misdirected medical tests. As a result of this experience, we will preface future research result return to the EHR with a preparatory e-mail, including screenshots. We also suggest small changes that EHR developers can make in subsequent software versions to allow slightly more customization in the return of research results.

\section{Clinical Relevance Statement}

The integration of genetic test results into the EHR, especially when originating from a research study, requires careful planning to avoid unintentional confusion. We identify lessons learned from a recent study and propose solutions for implementers and EHR vendors.

\section{Authors' Contributions}

The described work was conducted with input from all authors. L.V.R. and L.J.R.T. prepared the initial draft of the manuscript. All authors reviewed and approved the final manuscript.

\section{Protection of Human and Animal Subjects}

This work was reviewed and approved by the Northwestern University Institutional Review Board.
Funding

This study was funded by National Human Genome Research Institute, grant no.: U01HG8673.

Conflict of Interest

L.V.R., M.E.S., R.C., and J.S. report a patent related to the Ancillary Genomics System entitled "Generating Data in Standardized Formats and Providing Recommendations" that was submitted but is no longer being pursued. All other authors have no competing interests to disclose. Dr. Chisholm reports a patent GENERATING DATA IN STANDARDIZED FORMATS AND PROVIDING RECOMMENDATIONS that is no longer being pursued. Mr. Rasmussen reports a patent GENERATING DATA IN STANDARDIZED FORMATS AND PROVIDING RECOMMENDATIONS that is no longer being pursued. M.E.S. reports a patent GENERATING DATA IN STANDARDIZED FORMATS AND PROVIDING RECOMMENDATIONS that is no longer being pursued. J.S. reports a patent GENERATING DATA IN STANDARDIZED FORMATS AND PROVIDING RECOMMENDATIONS that is no longer being pursued.

\section{References}

1 Gottesman O, Scott SA, Ellis SB, et al. The CLIPMERGE PGx Program: clinical implementation of personalized medicine through electronic health records and genomics-pharmacogenomics. Clin Pharmacol Ther 2013;94(02):214-217

2 Rasmussen LV, Smith ME, Almaraz F, et al. An ancillary genomics system to support the return of pharmacogenomic results. J Am Med Inform Assoc 2019;26(04):306-310

3 Rosenman MB, Decker B, Levy KD, Holmes AM, Pratt VM, Eadon MT. Lessons learned when introducing pharmacogenomic panel testing into clinical practice. Value Health 2017;20(01):54-59

4 Starren J, Williams MS, Bottinger EP. Crossing the omic chasm: a time for omic ancillary systems. JAMA 2013;309(12):1237-1238

5 Whetton S, Georgiou A. Conceptual challenges for advancing the socio-technical underpinnings of health informatics. Open Med Inform J 2010;4:221-224

6 eMERGE Consortium. Electronic address: agibbs@bcm.edu eMERGE Consortium. Harmonizing clinical sequencing and interpretation for the eMERGE III Network. Am J Hum Genet 2019;105(03):588-605

7 Aronson S, Babb L, Ames DeMERGE Network EHRI Working Group. , et al; . Empowering genomic medicine by establishing critical sequencing result data flows: the eMERGE example. J Am Med Inform Assoc 2018;25(10):1375-1381

8 McCarty CA, Berg R, Waudby C, Foth W, Kitchner T, Cross D. Longterm recall of elements of informed consent: a pilot study comparing traditional and computer-based consenting. IRB 2015;37(01):1-5

9 Nishimura A, Carey J, Erwin PJ, Tilburt JC, Murad MH, McCormickJB. Improving understanding in the research informed consent process: a systematic review of 54 interventions tested in randomized control trials. BMC Med Ethics 2013;14:28 